\title{
FUZZY GENETIC ALGORITHM FOR SOLVING OPTIMIZATION PROBLEMS
}

\section{T. MUHAMEDIYEVA}

Tashkent University of Information Technologies named after Muhammad al-Khwarizmi

Amir Temurstreet, Tashkent, Uzbekistan

ABSTRACT
Considered a fuzzy genetic algorithm in which fuzzy rules for creating genetic operators with various properties and
fuzzy criteria for stopping the genetic search process are implemented using fuzzy logic tools. In this case, the
mathematical apparatus of the theory of fuzzy systems is used to encode, select the optimal parameters of genetic
algorithms, select the fitness function and stopping criterion, and create fuzzy genetic operators.
KEYWORDS:

Received: Jun 07, 2020; Accepted: Jun 27, 2020; Published: Jun 30, 2020; Paper Id.: IJMPERDJUN2020332

\section{INTRODUCTION}

The term "fuzzy genetic algorithms" was first coined by F. Herrera and M. Lozano [1-3]. According to the definition given in [4], a fuzzy genetic algorithm (GA) is a genetic algorithm in which some components are implemented using fuzzy logic tools.Such as fuzzy operators and fuzzy rules for creating genetic operators with various properties; systems of fuzzy logical control of the parameters of the genetic algorithm in accordance with accepted criteria;fuzzy criteria for stopping the genetic search process. The mathematical apparatus of the theory of fuzzy systems used in this case for coding, selection of optimal parameters of genetic algorithms, selection of the fitness function and stopping criterion, and creation of fuzzy genetic operators.

Currently, issues related to the theoretical and experimental study of genetic algorithms and the study of their properties in solving various classes of problems are very relevant.One of the serious problems that arise when using genetic algorithms is premature convergence.

Three main ways of eliminating premature convergence can be proposed: increasing the size of the population, using self-adaptive genetic operators and creating a "bank" of replaced individuals [5].

\section{Study of the Convergence of Genetic Algorithms to the Global Optimum}

Let's consider the problem of minimizing the function $f(X), X \in S$, where $S \subseteq R^{D}$ and $D$ - dimension of decision variables.Each decision is denoted as $X_{i, G}=\left[x_{1, i, G}, x_{2, i, G}, \ldots, x_{D, i, G}\right]$, where $i=1,2$, ..., $N P, N P$ is the size of the population and $G$ amount of current iteration.

Here is a description of the fuzzy genetic algorithm:

\section{Initialization of the Chromosome}

$j$-th parameter $X_{i, G}$ initialized as follows: 


$$
x_{i, j, G}=L_{j}+\operatorname{rndreal}(0,1) \cdot\left(U_{j}-L_{j}\right)
$$

where rndreal $(0,1)$ represents a uniformly distributed random number in the range $[0,1]$ and $L_{j}$ and $U_{j}$ represent the lower and upper bounds of the corresponding variable.

2. Crossover operator is applied to each pair of the target vector $X_{i, G}$ and corresponding mutant vector $V_{i, G}$ to generate a test vector $U_{i, G}$

$$
u_{j, i, G}= \begin{cases}v_{j, i, G}, & \text { if rndreal }(0,1) \leq c_{R} \text { or } j=j_{\text {rand }}, \\ x_{j, i, G}, & \text { in another case }\end{cases}
$$

where $j_{\text {rand }}$ is an integer randomly selected from the range $[1, D]$.

3. Mutation is applied independently to both subchromosomes, while a pair of genes in the chromosome are randomly selected, and their exchange between the subchromosomes occurs;

After the reproduction process, mutations occur.This operator is necessary for "knocking out" a population from a local extremum and prevents premature convergence.This is achieved due to the fact that a randomly selected gene in the chromosome changes.

Like crossingover, mutations can occur not only at one random point.You can choose to change several points in the chromosome, and their number may also be random.Mutations are also used with a change in a certain group of consecutive points at once.

4.Roulette wheel method is used as a selection, chromosomes survive with a probability proportional to the value of their fitness function.Operators of selection of individuals in a new population.To create a new population, various methods of selection of individuals can be used [6-9].

Truncation selection. When selecting by truncation, a population is used, consisting of both individuals-parents and individuals of descendants, sorted by increasing values of the fitness function of individuals. The number of individuals to be crossed is selected according to the threshold $T \in[0 ; 1]$. threshold determines what proportion of individuals, starting from the very first (most suitable), will take part in the selection.In principle, the threshold can also be set by a number greater than 1 , then it will simply be equal to the number of individuals from the current population admitted to the selection.Among individuals that fell "under the threshold", one is randomly selected and recorded in a new population.The process is repeated once, until the size of the new population becomes equal to the size of the original population. The new population consists only of individuals with high suitability, moreover, the same individual can occur several times, and some individuals having suitability above the threshold may not fall into the new population.Due to the fact that this strategy uses a sorted population, its working time may be large for a large population and also depend on the sorting algorithm[6-7].

Elite selection.An intermediate population is created, which includes both parents and their descendants.Members of this population are evaluated, and then $\mathrm{N}$ of the best (suitable) are selected from them, who will be included in the next generation.Often, this method is combined with others - they select, for example, $10 \%$ of "elite" individuals into a new 
population, and the remaining $90 \%$ - one of the traditional selection methods.Sometimes these $90 \%$ of individuals are created randomly, as when creating the initial population before starting the GA.Using the strategy of elitism is very useful for the effectiveness of the GA, since it does not allow the loss of better decisions.For example, if the population converged at a local maximum, and the mutation brought one of the lines to the global, then with the previous strategy it is very likely that this individual will be lost as a result of crossbreeding and no solution to the problem will be obtained. If elitism is used, then a good solution will remain in the population until an even better one is found[7-8].

Exclusion selection.In this selection, the choice of an individual in a new population depends not only on the size of its suitability, but also on whether an individual with a similar chromosome set is already in the formed population.Selection is carried out from among parents and their descendants.Of all individuals with the same fitness, preference is first given to individuals with different genotypes.Thus, two goals are achieved: firstly, the best solutions found that have different chromosome sets are not lost, and secondly, genetic diversity is constantly maintained in the population.In this case, crowding out forms a new population of more likely remote individuals, instead of individuals grouping around the current found solution. This method is most suitable for multi-extremal problems, while in addition to determining global extrema, it becomes possible to identify those local maxima whose values are close to global[9].

Boltzmann method, or annealing method (Bolzman selection).In this method, the probability of selection in a new population depends on the control parameter - temperature $T$.

GA parameters.Crossover probability is usually chosen quite high: $80-95 \%$.However, in some tasks, the best result is achieved with crossing over with a probability of $60 \%$. The probability of mutation should be low: $0.5-1 \%$.

Oddly enough, a very large population size usually does not lead to good results (the convergence rate of the algorithm does not increase).The optimal population size is 20-30 individuals, however, in some tasks, as researchers write, 50-100 individuals are required.Studies show that the optimal population size depends on the size of the code lines (chromosomes).So, for an algorithm with 32-bit chromosomes, the population size will be larger than for an algorithm with 16-bit chromosomes.

Mostly they use roulette selection, but sometimes it is better to apply selection with truncation.There are many other methods that change the selection parameters during the entire GA.Elitism is applied if other methods are not used that preserve the good solution found.The choice of coding method is determined by the task and the size of the search object [9-12].

Selection operator is described as follows:

$$
X_{j, G+1}= \begin{cases}U_{i, G}, & \text { if } f\left(U_{i, G}\right) \leq f\left(X_{i, G}\right), \\ X_{i, G}, & \text { in another case. }\end{cases}
$$

We give additional definitions for the convergence of the genetic algorithm:

Let $x_{t}: t \geq 0 \mathrm{~s}$ a sequence of chromosome populations generated by a genetic algorithm, and let be the corresponding value of the best chromosome in a population at a time $t$. Genetic algorithm has full convergence to the global optimum $f^{*}$ optimization tasks defined by function $f: x \rightarrow R$, if a non-negative random sequence 
$D_{t}=f^{*}-F_{t}$ converge all the way to 0.

In general, one step of the genetic algorithm can be represented as follows:

$$
\begin{aligned}
& \left(x_{1}^{\prime}, \ldots, x_{n}^{\prime}\right)=\operatorname{sel}\left(x_{1}, \ldots, x_{n}\right), \\
& \left(x_{1}^{\prime \prime}, \ldots, x_{n}^{\prime \prime}\right)=\operatorname{cross}\left(x_{1}^{\prime}, \ldots, x_{n}^{\prime}\right), \\
& \left(x_{1}^{\prime \prime \prime}, \ldots, x_{n}^{\prime \prime \prime}\right)=\operatorname{mut}\left(x_{1}^{\prime \prime}, \ldots, x_{n}^{\prime \prime}\right),
\end{aligned}
$$

where $\left(x_{1}, \ldots, x_{n}\right) \in x^{n}$ - current chromosome population; $\left(x_{1}^{\prime}, \ldots, x_{n}^{\prime}\right)$ - chromosome population resulting from selection; $\left(x_{1}^{\prime \prime}, \ldots, x_{n}^{\prime \prime}\right)$ - cross-breeding chromosome population; $\left(x_{1}^{\prime \prime \prime}, \ldots, x_{n}^{\prime \prime \prime}\right)$ - mutation chromosome population.

- In [5], it was proved that evolutionary algorithms converge both on average and completely to the global optimum under the following conditions:

- Each chromosome in a population can be changed to an arbitrary other chromosome in one single mutation with probability $p>0$.

- The best chromosome in a population survives in every generation with a probability $p=0$.

- Formally, these conditions can be represented as:

$$
\begin{aligned}
& \forall x, y \in X \quad p\{y=\operatorname{mut}(x)\} \geq \delta_{m}>0, \\
& p\left\{V_{n}^{*}\left(\operatorname{sel}\left(x_{1}, \ldots, x_{k}\right)\right)=V_{k}^{*}\left(x_{1}, \ldots, x_{k}\right)\right\}=1,
\end{aligned}
$$

where $V_{i}^{*}$ operator returning the best chromosome from the chromosomes of a population.

If condition 1 is valid, it can be shown that the evolutionary algorithm converges to a global optimum in a finite number of steps with probability $p=1$, regardless of its initialization, but can converge if it cannot be guaranteed that the optimum will indeed remain in the population after it has been found.If condition 2 is also true, then it can be shown that the evolutionary algorithm converges to a global optimum.

Thentheexpressions

$$
\begin{aligned}
& p\left\{V_{n}^{*}\left(\operatorname{sel}\left(x_{1}, \ldots, x_{k}\right)\right)=V_{k}^{*}\left(x_{1}^{\prime}, \ldots, x_{n}^{\prime}\right)\right\}=1, \\
& p\left\{V_{n}^{*}\left(\operatorname{cross}\left(x_{1}^{\prime}, \ldots, x_{n}^{\prime}\right)\right)=V_{k}^{*}\left(x_{1}^{\prime \prime}, \ldots, x_{n}^{\prime \prime}\right)\right\}=1,
\end{aligned}
$$

correctly describe condition 2 with respect to GA.

We show that the genetic algorithm converges completely and on average to the global optimum. To do this, we show that conditions 1 and 2 are satisfied.Mutation and crossing operators can make changes to chromosomes, the use of which helps to find the optimum. 
Let bit strings of chromosomes be represented by a vector $\{0,1\}^{L}$. If when comparing a string of bits of length $(L-c)$ in the chromosome represents the optimum, therefore, with the bits of the chromosomes and does not coincide with the optimum.Then the probability of the mutation and crossing operator to achieve a global optimum in one step is $P_{c}^{(L)}=\frac{c !}{L_{c}} \cdot \frac{1}{L}$. Here is a favorable number of choices $c !$ is the number of permutations from elements from the possible number of choices $L_{c}$. This probability should be multiplied by the probability that the mutation operator randomly changes the bits, i.e.on $1 / L$, which is the probability that $c=r$, where $r$-randomly selected number of bits that mutate.

If the chromosome is represented by a vector $\{0,1, \ldots, k-1\}^{L}$, each element of which is taken from the alphabet from $k$ elements, then the probability that each of the numbers that will be susceptible to mutation and crossover will be a digit of the optimal chain is

$$
P_{c}^{(L)}=\frac{c !}{L_{c}} \cdot \frac{1}{(k-1)^{c}} \cdot \frac{1}{L}
$$

If $P_{c}^{(L)}$ always positive, then condition 1 is satisfied.It should be noted that there is also a probability $p_{s}>0$ of the fact that the optimum was accidentally introduced into the population by the selection operator, which should be considered, although the probabilistic $P_{c}^{(L)}$ enough to show that condition 1 is fulfilled.

To show that condition 2 is also fulfilled, it is necessary to take into account all the operators acting on the population, and also to show that none of them leads to a loss of the optimal solution if it is found. Let us analyze the operators of the genetic algorithm.

Selection operator, after getting rid of the least suitable chromosomes, replenishes the population with new chromosomes, so the optimum can not beat lost.

Mutation and crossing operator acts only on the chromosomes of a population, without touching the best chromosome in the population.Therefore, these operators also do not lose optimum. Thus, the above reasoning shows that condition 2 is satisfied. Consequently, the genetic algorithm converges if the mutation, cross and selection operators satisfy condition 1, and condition 2 also holds.

We show that the genetic algorithm may not completely converge to the global optimum, regardless of its initialization, if the mutation operator changes individual bits and a non-elite version of the crossover operator is used.

The fulfillment of condition 1 was shown above, according to which the genetic algorithm reaches the optimum, provided that it uses the mutation, cross, and (or) selection operators.It is necessary to show the fulfillment of condition 2 , which is a necessary but not sufficient condition for convergence.

To show that the genetic algorithm does not completely converge to a global optimum, it suffices to show that each time an optimum is found, there will be the next generation of chromosomes, which the genetic algorithm does not have an optimum in the population.If there is a probability, even a very small one, that the genetic algorithm will go out of the optimum, having found at another moment of time another optimal solution, then this means that the genetic algorithm 
does not completely converge with probability 1 . This probability is also guaranteed by the non-elitism of the selection operator in the sensethat a chromosome representing optimism at reaching age through generations will be changed in a population.Selection operator will create a population consisting of copies of optimal solutions. $x_{1}$ and from non-optimal solutions $x_{2}$. Since the mutation operator always changes at least one bit of the chromosome optimal solutions will not reach global optimums.On the other hand, each non-optimal solution can become optimal in the next step with a probability such that $p \leq p_{d=1}$, where $p_{d=1}$ is the probability of reaching an optimum from the most probable state in steps $d=1$.

These considerations prove the following statement.

Statement.The convergence analysis of the genetic algorithm is based on two conditions:

- As a result of mutation and crossing, it is possible to achieve the optimal state from the non-optimal one in one step;

- Once the optimal condition is found, it will remain in the population and will not be lost.

\section{COMPUTATIONAL EXPERIMENT}

In this paper, 3 test functions are used to test methods and conduct experiments on methods.All test functions may have a different number of parameters (d).Therefore, it makes sense to run an algorithm to optimize a function, first with a small d (for example, 10 or 20), and then with $d=50,100,200, \ldots$ This will make it possible to check the scalability of the algorithm.

\subsection{LangermannFunction:}

$$
f(x)=\sum_{i=1}^{m} c_{i} \exp \left(-\frac{1}{\pi} \sum_{j=1}^{d}\left(x_{j}-A_{i j}\right)^{2}\right) \cos \left(\pi \sum_{j=1}^{d}\left(x_{j}-A_{i j}\right)^{2}\right) .
$$

Recommended Variables for $\mathrm{d}=2: \mathrm{m}=5, \mathrm{c}=(1,2,5,2,3)$ and

$$
A=\left(\begin{array}{ll}
3 & 5 \\
5 & 2 \\
2 & 1 \\
1 & 4 \\
7 & 9
\end{array}\right) .
$$

Domain:

$x_{i} \in[0,10], i=1, \ldots, d$.

Genetic algorithm:

$d=2$, number of finds of the global minimum $84 \%$, the number of calculations of the objective function no more than 1250in GA. Number of finds of the global minimum $92 \%$, the number of calculations of the objective function no 
more than 530 in fuzzy GA.

\section{CONCLUSIONS}

Proposed fuzzy crossing-over and mutation operators can be effectively applied to solve fuzzy optimization problems.Test problems are solved on the basis of the genetic algorithm, the classical gradient method and Newton's method, a comparative analysis of the results of solving these problems is carried out. It is shown that the genetic algorithm is a universal algorithm for finding the optimum of functions of any complexity, but when constructing simple functions, this algorithm loses in speed to the gradient method. It is also determined that the Newton method is optimal for the Langermann and Levy functions, and the genetic algorithm for the Rastrigin functions.

\section{REFERENCES}

1. Herrera F., Lozano M., Verdegay J. 1995 Tackling fuzzy genetic algorithms. In Winter. Genetic Algorithms in Engineering and Computer Science, 167

2. Herrera F., Lozano M.1998 Fuzzy Genetic Algorithms: Issues and Models.Technical Report DECSAI-98116, Dept. of Computer Science and AI, University of Granada,

3. Anusuya, V., and R. Kavitha. "Roulette ant wheel selection (RAWS) for genetic algorithm-fuzzy shortest path problem." International Journal of Mathematics and Computer Applications Research 5 (2015): 1-14.

4. Herrera F., Lozano M. 2003 Fuzzy Adaptive Genetic Algorithms: design, taxonomy, and future directions.Soft Computing 7, 545

5. Galantucci L.M., Percoco G., Spina R. 2004 Assembly and Disassembly Planning by using Fuzzy Logic \& Genetic Algorithms.International Journal of Advanced Robotic Systems. 167

6. GolubinA.V., TarasovV.B. 2005Nechetkiyegeneticheskiyealgoritmi.Mejdunarodniyenauchno-texnicheskiyekonferensii. 39

7. Lin, Zhi-Ping, Her-Shing Wang, and Shao-Jyun Tsai. "The Intelligent Charging Path Planning for Electric Vehicle." International Journal of Computer Networking, Wireless and Mobile Communications (IJCNWMC) ISSN (P) (2016): $2250-1568$.

8. Lozano M., Herrera F., Krasnogor N., Molina D. 2004 Real-Coded Memetic Algorithms with Crossover Hill-Climbing. Evolutionary Computation.12, 273

9. Alvarez A. 2000 Forecasting the SST Space-Time Variability of the Alboran Sea with Genetic Algorithms. Geophysical Research Letters.

10. Mahela, Om Prakash, and Sheesh Ram Ola. "Optimal capacitor placement for loss reduction in electric transmission system using genetic algorithm." TJPRC-International Journal of Electrical and Electronics Engineering Research 3.2 (2013): 59-68.

11. RutkovskayaD., PilinskiyM., RutkovskiyL. 2004Neyronniyeseti, geneticheskiyealgoritmiinechetkiyesistemi.Goryachayaliniya Telekom, 452

12. Gen M., Cheng R. 1997 Genetic algoritms and engineering design. John Wiley\&Sons.352

13. Muhamediyeva D.T. and Niyozmatova N.A. 2019 Problems of constructing models of intellectual analysis ofstates of weakly formalizable processes.Journal of Physics: 1210

14. Devi, K. Devaki, K. Satish Babu, and K. HEMACHANDRA REDDY. "Optimization of Cutting Force And Tool Temperature Using Ann Based Multi Objective Genetic Algorithms In Turning Heat Treated Beryllium Copper Alloy." International 
Journal of Mechanical and Production Engineering Research and Development (IJMPERD) ISSN (P) (2016): 2249-6890.

15. Muhamediyeva D.T. and Niyozmatova N.A. 2019 Approaches to solving the problem of fuzzy parametric programming in weakly structured objects.Journal of Physics.1260

16. Muhamediyeva D.T. and Sayfiyev J. 2019 Approaches to the construction of nonlinear models in fuzzy environment.Journal of Physics.1260 